\title{
Tratamento cirúrgico da lesão isolada de óstio coronário esquerdo
}

\author{
Ricardo Ribeiro DIAS * , Altamiro Ribeiro DIAS *, Marcelo B. JATENE * , Adib D. JATENE *
}

DIAS, R. R.; DIAS, A. R.; JATENE, M. B.; JATENE, A. D. - Tratamento cirúrgico da lesăo isolada de óstio coronário esquerdo. Rev. Bras. Cir. Cardiovasc., 10 (3): 154-159, 1995.

RESUMO: A experiência do InCór no tratamento cirúrgico da lesão isolada de óstio coronário esquerdo é de 11 pacientes, operados no período de janeiro de 1984 a julho de 1994 . Oito pacientes eram do sexo feminino $e 3$ do sexo masculino, todos de cor branca, com idades de 39 a 68 anos (média de 53 anos). Dois pacientes eram assintomáticos, 2 apresentavam angina instável e os demais eram anginosos crônicos. Todos tinham lesāo isolada de óstio coronário esquerdo de cerca de $90 \%$. Os 11 pacientes foram operados com perfusāo extracorpórea, através de aortotomia transversa posterior prolongada para artéria coronária esquerda até sua bifurcaçăo. A artéria coronária esquerda era normal em todos os casos. Fez-se a ampliaçăo do óstio com enxerto de vela safena do paciente em 8 casos e com enxerto de pericárdio bovino em 3 casos. A biopsia de aorta realizada próximo á obstruçāo apresentou infiltraçāo mucóide inespecífica. Dez pacientes evoluíram bemno pós-operatório imediato. Um paciente submetido a cinecoronariografia no oitavo dia de pósoperatório, dado como normal, evoluiu com morte súbita cerca de 7 horas após o procedimento. A necropsia revelou trombo de $8 \mathrm{~mm}$ de diâmetro ao nivel da ampliaçăo e ateromatose de ramos coronários. Ocorreu outro óbito, dado como hepatite, nove meses após a operaçāo. Os demais pacientes apresentam-se clinicamente bem, num período de seis meses a dez anos de evoluçăo. Os resultados obtidos sugerem que a modalidade técnica cirúrgica empregada para a ampliaçăo do óstio coronário esquerdo é boa, com mortalidade hospitalar aceitável $(9 \%)$.

DESCRITORES: Óstio coronário, cirurgia.

\section{INTRODUÇÃO}

A lesäo isolada de óstio coronário esquerdo é entidade patológica rara, de sintomatologia variável e prognóstico reservado, se não tratada no seu devido tempo 5,7 .

O tratamento habitualmente empregado consiste em ponte aorto-coronária com enxerto de veia safena ou artéria torácica interna. Porém, a técnica cirúrgica que visa à abordagem direta do óstio da artéria coronária nāo é nova. LONGMIRE et alii ${ }^{12}$, em 1948, seguidos por SABISTON et alii ${ }^{16} \dot{\theta}$ EFFLER et alii ${ }^{6}$, em 1965 , já preconizavam a am- pliaçăo do óstio e tronco da artéria coronária para lesőes estenosantes, principalmente associadas a lesăo aterosclerótica e aortite sifilítica. Tal técnica foi momentaneamente abandonada pela alta mortalidade imediata apresentada (maior que $45 \%$ ). Em 1982, HITCHCOCK et alii ${ }^{10}$ relatam sua experiência e atribuem seus resultados positivos principalmente em funçăo da evolução das técnicas de proteçāo miocárdica durante o ato cirúrgico.

O objetivo deste trabalho é apresentar a experiência cirúrgica do InCór na abordagem direta do óstio da artéria coronária esquerda e seus resultados em seguimento de até dez anos.

Trabalho realizado no Instituto do Coraçăo do Hospital das Clínicas da Faculdade de Medicina da Universidade de Săo Paulo. Săo Paulo, SP, Brasil. Apresentado ao $22^{\circ}$ Congresso Nacional de Cirurgia Cardlaca. Brasillia, DF, 30 de março a $1^{\circ}$ de abril, 1995.

* Do Instituto do Coraçã̌o do Hospital das Clinicas da Faculdade de Medicina da Universidade de Săo Paulo.

Endereço para correspondència: Ricardo Ribeiro Dias. Av. Dr. Enéas de Carvalho Aguiar, 44. Divisāo Cirúrgica. CEP: 05403-000 Sāo Paulo, SP, Brasil. 
DIAS, R. R.; DIAS, A. R.; JATENE, M. B.; JATENE, A. D. - Tratamento cirúrgico da lesão isolada de óstio coronário esquerdo. Rev. Bras. Cir. Cardiovasc., $10(3): 154-159,1995$.

\section{CASUISTICA E MÉTODOS}

Foram estudados 11 pacientes operados no periodo de janeiro de 84 a julho de 94 , com tempo de seguimento variando entre seis meses e 10 anos; 8 pacientes eram do sexo feminino e 3 do masculino. Todos da raça branca. As idades variaram de 39 a 68 anos, com média de 53 anos.

Clinicamente, 2 pacientes encontravam-se assintomáticos, tendo-se diagnosticado lesão a partir de exames de rotina. Dois pacientes apresentavam angina instável e os demais 7 eram anginosos crônicos (apresentando, 1 destes, dispnéia a médios esforços secundária a estenose mitral associada).

Para todos os pacientes, a investigaçăo cinecoronariográfica revelou lesão isolada do óstio coronário esquerdo de cerca de $90 \%$.

\section{TÉCNICA CIRÚRGICA}

Todos os pacientes foram operados por esternotomia mediana, perfusão extracorpórea, hipotermia moderada $\left(28^{\circ}-30^{\circ} \mathrm{C}\right)$ e soluçăo cardioplégica de St. Thomas I em toda a série. Realizou-se, em todos, aortotomia transversal posterior direcionada para óstio coronário esquerdo, seccionando-se lesão suboclusiva ostial (Figura 1), estendendo-se distalmente até a bifurcaçāo da coronária esquerda. Em seguida, confeccionou-se a ampliaçäo de artéria coronária, óstio e aorta circunjacente com enxerto de veia safena em 8 pacientes e com enxerto de pericárdio bovino fixado em glutaraldeído nos demais (Figura 2). Após a ampliação, o óstio esquerdo passou a aceitar sonda exploradora de 4 a $5 \mathrm{~mm}$ em todos os pacientes (1 dos pacientes foi submetido a plástica de valva mitral, sem intercorrências).

\section{AVALIAÇÃO ANATOMOPATOLÓGICA}

As lesōes ostiais eram circulares e suboclusivas em todos os casos. Não havia placas ateroscleróticas, sendo o tecido de consistência firme. Ultrapassada a lesão ostial, a artéria coronária era de aspecto macroscópico normal. Estas características sugerem que o acometimento patológico $\dot{e}$, na realidade, da aorta e năo da coronária. As biopsias de aorta, estudadas com coloraçāo especifica, demonstraram infiltraçăo mucóide inespecífica em todos os 8 casos (três amostras não foram recuperadas).

\section{RESULTADOS}

Dez pacientes apresentaram evoluçăo pós-operatória imediata sem intercorrências.
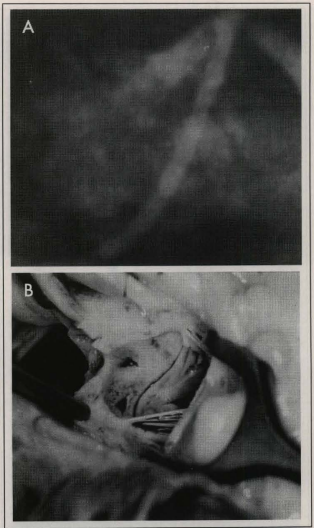

Fig. 1 - A) Cinecoronariografia pró-operatóna. Observa-se leaâo suboclusiva de ostio coronário esquerdo. B) Aorta aberta posteriormente, com exposiçăo do óstio coronário esquerdo subociuido (aortotomia interrompida aonivel de antéria coronária esquerda).

Cerca de duas semanas após a operação, todos os pacientes foram submetidos a avaliação cinecoronariográfica. Todos apresentavam excelente ampliação do óstio coronário esquerdo (Figura 3 ). Um paciente, sete horas após o estudo cinecoronariográfico pós-operatório, apresentou dor precordial intensa, seguida de fibrilaçāo ventricular e parada cardiorespiratória irreversivel às manobras habituais de ressuscitação. A necropsia demonstrou presença de trombo de cerca de $8 \mathrm{~mm}$ na regiăo da ampliaçāo do óstio, além de lesōes ateroscleróticas significativas $(70 \%$ em ramo interventricular anterior proximal e $60 \%$ em ramo circunflexo proximal), subestimadas no estudo pré-operatório (Figura 4).

Dez pacientes receberam alta hospitalar em boas condiçőes. Nove meses após a alta, outro 

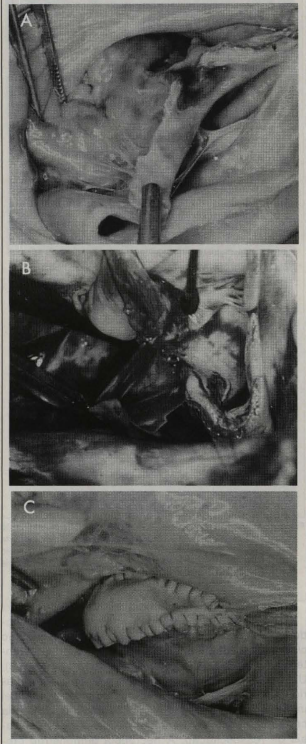

Fig. 2 - A) Aorta aberta posteriormente, observando-se secção de óstio coronário esquerdo, incisado até a bifurcaçăo da artéria coronária esquerda. B) Observa-se enxerto de pericárdio bovino sendo suturado distalmente, ao nivel da bifurcaçẫo da artéria coronária esquerda. A sutura contínua progride em direçăo ao dstio que será ampliado, C) Observa-se o enxerto de pericárdio bovino completamente suturado. Este enxerto tem forma eliptica, com maior diámetro transverso ao nivel do óstio. Na seqũência, a aortotomia posterior suturada.

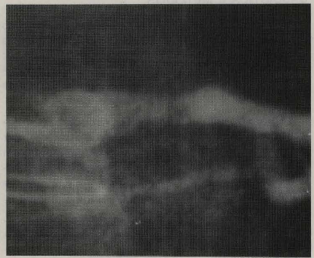

Fig. 3 - Cinecoronariografia pós-cperatória. Observa-seo óstio coronário esquerdo com boa ampliaçâo.

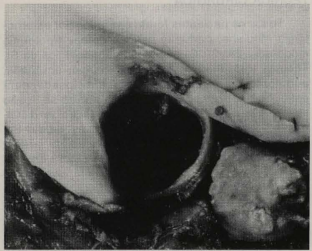

Fig. 4 - Peça anatómica do paciente que faleceu no pós-operatório imediato, 7 horas após o estudo cinecoronariográfico de controle. Aorta aberta e na regiāo correspondente ao seio coronário esquerdo pode-se visibilizar parcialmente o trombo que oclui a regiáo do óstio.

paciente veio a falecer com quadro relatado, pela familia, como hepatite.

A mortalidade imediata foi de $9 \%$ e a tardia de $10 \%$ (em seguimento de seis a dez anos)

Os 9 pacientes restantes apresentam-se assintomáticos quanto à angina. A paciente submetida a plástica da valva mitral encontra-se em insuficiência cardiaca congestiva classe funcional II em função de reestenose valvar, reestudada angiograficamente, com seis anos de pós-operatório, apresenta óstio coronário esquerdo ampliado sem qualquer lesão estenosante. Outras 2 pacientes, assintomáticos, que, em exames de rotina, apresentaram teste ergométrico positivo, foram reestudadas angio- 
graficamente (cinco e sete anos de pós-operatório) e não foi constatada qualquer lesão obstrutiva, nem alteração de função de ventrículo esquerdo.

\section{COMENTÁRIOS}

A intervençăo direta em lesões obstrutivas de óstio ou de tronco da artéria coronária esquerda náo é recente 5 . Porém, foi somente a partir da experiência cirúrgica de HITCHCOCK et alii ${ }^{10}$ que se iniciou, definitivamente, a abordagem das lesóes de óstio por ampliaçāo. A via de acesso na abordagem de aorta e artéria coronária esquerda e o tipo de enxerto utilizado para a ampliaçao local ainda não apresenta consenso na literatura, porém há tendência, nos trabalhos mais recentes, de se utilizar o pericárdio bovino para a ampliaçăo e sugestão para acesso anterior para melhor exposição da artéria coronária esquerda, através de ampla dissecção da aorta e tronco pulmonar $4,9,13$.

O critério de seleçāo dos pacientes com lesão de óstio a serem submetidos à ampliaçāo também sofreu mudanças. Inicialmente, HITCHCOCK et alii ${ }^{10}$ preconizavam que só havia indicação para tal procedimento cirúrgico para aqueles pacientes com angina aos mínimos esforços, lesảo calcificada de óstio maior que $50 \%$, ventrículo esquerdo com função preservada e sem infarto prévio. DEUVAERT et alii ${ }^{3}$ ampliam as indicaçōes para os casos de angina instável e para pacientes com infarto prévio de pequeno tamanho. Mais recentemente, DION et alii ${ }^{4}$ relatam somente não realizar a ampliação de óstio nos casos em que a lesăo ultrapassa a bifurcaçăo da artéria coronária, ou para artérias com sinal de calcificação no estudo cinecoronariográfico.

No InCór, dos 11 pacientes submetidos à ampliaçāo de óstio da artéria coronária esquerda, 2 pacientes apresentaram angina instável, todos com lesão de óstio de cerca de $90 \%$, todos com função ventricular preservada e nenhum com história prévia de infarto do miocárdio. Apenas 1 paciente evoluiu para óbito no pós-operatório imediato, sete horas após cinecoronariografia que revelara excelente ampliação da lesão de óstio, sugerindo que, talvez, as lesōes subestimadas em ramo interventricular anterior e ramo circunflexo ou que a manipulação provocada pelo cateterismo influenciaram, de alguma forma, a trombose local e éxito letal do paciente.

Nossa estatística revela mortalidade intra-hospitalar de $9 \%$. No seguimento tardio de seis meses a dez anos, nenhum paciente apresentou recidiva clínica de angina ou reestenose da lesão primária detectáveis. O único óbito constatado nesta fase deveu-se a quadro de hepatite aguda e não sabemos se é possível associar o quadro ao procedimento cirúrgico, por falta de dados.

O fator etiológico associado à lesão de óstio identificado em nossa amostragem năo difere muito da literatura; infiltração mucóide inespecífica, caracterizando acometimento intrínseco da aorta com comprometimento de óstio. Porém, em nenhum dos casos foram caracterizados fatores etiológicos específicos, tais como arterite de Takayasu, sifilis, hipercolesterolemia familial congênita, membrana congênita de óstio, iatrogenia durante procedimentos angiográficos 1, 2, 11, 14, 15, 17 .

Em funçāo de avaliação da experiência adquirida, concluímos que a técnica cirúrgica empregada é reprodutivel, a indicaçăo deve ser precisa, limitada para pacientes com lesão severa (cerca de $70 \%$ ou mais) e isolada de óstio coronário esquerdo, independente do tipo de angina. A mortalidade constatada é aceitável, o que nos estimula a continuar com a utilizaçăo do método. 
DIAS, R. R.; DIAS, A. R.; JATENE, M. B.; JATENE, A. D. - Tratamento cirúrgico da lesāo isolada de óstio coronário esquerdo. Rev. Bras. Cir. Cardiovasc., $10(3): 154-159,1995$.

RBCCV $44205-273$

DIAS, R. R.; DIAS, A. R.; JATENE, M. B.; JATENE, A. D. - Surgical management of left coronary ostial lesion. Rev. Bras. Cir. Cardiovasc., 10 (3): 154-159, 1995.

ABSTRACT: The experience of the Heart Institute of the University of Sāo Paulo with surgical management of left coronary ostial lesions was made of 11 patients. They were operated on from January 1984 to July 1994. Eight of them were female, 3 were male. They were of white race. The ages ranged from 39 to 68 years old (mean 53). Two patients were asymptomatic, 2 were with unstable angina and the others with chronic angina. All of them had ostial stenosis of $90 \%$ or more. They were operated on with cardio-pulmonary bypass, posteria oblique aortotomy prolonged through the left main coronary artery. The ostioplasty was made with saphenous vein patch in 8 cases and with gluteraldehyde preserved xenopericardial patch in 3 cases. The aortic root biopsies showed inespecif mucoid intiltrations in all studied cases. All patients were angiografically studied 2 weeks after surgery. The ostial angioplasty were opened widely in all cases. One patient died 7 hours after angiografic study, and the necropy revealed a large thrombus of $8 \mathrm{~mm}$ inside of the left ostioplasty. One patient died 9 months later, with a diagnosis of hepatitis. The other 9 patients have been followed up from 6 months to 10 years, and are all free of angina and doing well. The results take us to believe that the surgical technique is applicable to this kind of stenosis, with good results.

DESCAIPTORS: Coronary ostium, surgery.

\section{REFERÊNCIAS BIBLIOGRÁFICAS}

1 BARNER, H. B.; REESE, J.; STANDEVEN, J.; McBRIDE, L. R.; PENNINGTON, G.; WILLMAN, V. L.; KAISER, G. C. - Left coronary ostial stenosis: comparison with left main coronary artery stenosis. Ann. Thorac. Surg., 47: 293-296, 1989.

BRENNER, W. I.; MIRANTE, K.; MAHRER, P. R. - Direct ostioplastic of the left main coronary artery for isolated nonarteriosclerotic ostial stenosis. $J$. Thorac. Cardiovasc. Surg., 103: 168-70, 1992.

DEUVAERT, F. F; PAEPE, J.; NOOTEN, G.: PEPERSTRAETE, D.; PRIMO, G. - Transaortic saphenous patch angioplasty for left main coronary artery stenosis. J. Cardiovasc. Surg., 29: 610-613, 1988.

DION, R. \& PUTS, J. P. - Bilateral surgical ostial angioplasty of the right and left coronary arteries. $J$. Thorac. Cardiovasc. Surg., 102: 643-645, 1991.

DION, R.; VERHELST, R.; MATTA, A.; ROUSSEAU, M.; GOENEN, M.; CHALANT, C. - Surgical angioplasty of the left main coronary artery. $J$. Thorac. Cardiovasc. Surg., 99: 241-250, 1990.

6 EFFLER, D. B.: SONES. F. M.; FAVALORO, R.; GROVES, L. K. - Coronary endarterotomy with patchgraft reconstruction: clinical experience with 34 cases. Ann. Surg., 162: 590-601, 1991.

7 ENG, J.; BETON, D. C.; LAWSON, R. A. M.; MOUSSALLI, H.; NAIR, U. R.; RAHMAN, A. N. Coronary ostial stenosis: surgical considerations. Internat. J. Cardiol., 30: 285-288, 1991.

8 GOMBERG, J.; KLEIN, L. W.; SEELAUS, P.; PARR, G. V. S.; AGARWAL, J. B.; HELFANT, R. H. - Surgical revascularization of the left main coronary artery stenosis: determinants of perioperative and long-term outcome in the 1980s. Am. Heart J., 116: 440-446, 1988.

9 GOSH, P. K. - Coronary ostial reconstruction: technical issues. Ann. Thorac. Surg., 51: 673-675, 1991.

10 HITCHCOCK, J. F:; MEDINA, R.; JAMBROES, G. Angioplasty of the left main coronary artery for isolated left main coronary artery disease. J. Thorac. Cardiovasc. Surg., 85: 880-884, 1983.

11 JOSA, M.; DANIELSON, G. K; WEIDMAN, W. H. EDWARDS, W. D. - Congenital ostial membrane of left main coronary artery. $J$. Thorac. Cardiovasc. Surg., 81: 338-346, 1981.

12

MATSUDA, H.; MIYAMOTO, Y.; TAKAHASHI, T.; KADOBA, ; NAKANO, S.; SANO, T. - Extended aortic and left main coronary angioplasty with a single pericardial patch in a patient with Williams Syndrome. Ann. Thorac. Surg, 52: 1331-1333, 1991.

14 PENNINGTON, D. G.; DINCER, B.; BASHITI, $H$. BARNER, H. B.; KAISER, G. C.; TYRAS, D. H.; CODD, J. E.; WILLMAN, V. L. - Coronary artery stenosis following aortic valve replacement and intermittent intracoronary cardioplegia. Ann. Thorac. Surg., 33: 576-584, 1982. 
DIAS, R. R.; DIAS, A. R.; JATENE, M. B.; JATENE, A. D. - Tratamento cirúrgico da lesăo isolada de óstio coronário esquerdo. Rev. Bras. Cir. Cardiovasc., $10(3): 154-159,1995$.

RIBEIRO, P.: SHAPIRO, L. M.; GONZALEZ, A.; THOMPSON, G. R.; MOAKLEY, C. M. - Cross sectional echocardiographic assesment of the aortic root and coronary ostial stenosis in familial hypercholesterolaemia. Br. Heart J., 50: 432-437, 1983.

16 SABISTON, D. C.: DURHAM, EBERT, P. A.; FRIESINGER, G. C.; ROSS, R. S.; SINCLAIR-
SMITH, B. - Proximal endarterectomy, arterial reconstruction for coronary occlusion at aortic origin. Arch. Surg. 91: 758-764, 1965.

17 SWAHN, E; KARLSSON, J. E.; FRANSSON, S. G.; LINDSTROM, F.; NYLANDER, E.; STAHL, E. Coronary ostial stenosis operated on by patch technique in a young woman with Takayasu's arteritis and angina pectoris. Eur. Heart J., 14: 1150-1151, 1993. 